\title{
Lost in Translation: Sesgos y la información en la pandemia de COVID-19
}

\section{Lost in Translation: Biases and information in the COVID-19 pandemic}

David Torres P. MScㄹ, Juan Pablo Valenzuela²

\begin{abstract}
The COVID-19 pandemic has produced an endless stream of information in relation to data, analysis and projections of all aspects of this disease. One of the characteristics of scientific information is that it is full of uncertainties, which are not correctly disclosed to the general population. In this essay, we describe the main cognitive biases that cause people to modify their perception of risk, where the most important are memory, novelty, and emotionality or affection. In addition, we review frequent errors that have been made in terms of collection, dissemination and analysis of information by scientific communicators, experts in other areas and the media in general. Ideally, these biases and confounders should be known to all who participate in the flow of information on the health problems of this pandemic, in order to integrate the uncertainty inherent in the data and the critical analysis of the information received.
\end{abstract}

\section{RESUMEN}

La pandemia COVID-19 ha producido un caudal interminable de información en relación a datos, análisis y proyecciones de todos los aspectos de esta enfermedad. Una de las características de la información científica es que está llena de incertidumbres, las que no se divulgan correctamente a la población general. En este ensayo, hacemos una descripción de los principales sesgos cognitivos que hacen que las personas modifiquen su percepción de riesgo donde los más importantes son el de memoria, de novedad y emocionalidad o afecto. Además,

\section{Key words:}

Bias, confounding

factors, communication

\section{Palabras clave:}

Sesgo, factores de confusión, comunicación

\footnotetext{
'Médico Anestesiólogo; MSc Epidemiología Clínica. Universidad de los Andes, Chile. Clínica Santa María. Outcomes Research. 2Psicólogo.
}

Fecha de recepción: 12 de abril de 2020

Fecha de aceptación: 12 de abril de 2020

\section{ORCID}

https://orcid.org/0000-0003-2908-6365

Correspondencia:

Dr. David Torres, MSc

Email: dtorresp@gmail.com 
revisamos errores frecuentes que se pueden cometer en términos de recolección, difusión y análisis de la información de parte de comunicadores científicos, expertos en otras áreas y medios de comunicación en general. Idealmente, estos sesgos y confundentes deben ser conocidos por todos los que participan en el flujo de información de los problemas de salud de esta pandemia, de manera de poder integrar la incertidumbre inherente a los datos y el análisis crítico de la información que se recibe.

\section{Introducción}

Si se requiriera definir en una palabra el desarrollo actual de la literatura científica sobre la reciente pandemia del COVID-19, sería "incertidumbre". Esto resulta algo paradójico al observar en redes sociales y distintos canales de comunicación masivos, formales e informales bastantes "certezas"; desde las tasas de contagio a nivel país, análisis a partir de tasas de letalidad brutas, modelos computacionales de proyecciones y estimaciones de casos, modelos teóricos sobre "aplanar la curva", la eficiencia de medidas de control poblacional como la cuarentena total, medidas individuales como la vacuna BCG y de intervenciones específicas a nivel país que podrían ayudar a solucionar los problemas originados por la letalidad, el acceso a servicios de salud y el control de este brote epidémico.

La mayoría de estas afirmaciones y análisis parecieran desconocer que suelen estar basadas en datos que evidencian numerosas inconsistencias, supuestos y limitaciones, cargados de sesgos y confundentes, lo que finalmente desemboca en una capacidad reducida de entregar información suficientemente confiable. Además, de la calidad del origen de los datos y su consecuente análisis, debemos incorporar la interpretación que realizan las personas al recibir estas afirmaciones e información nueva, tanto desde emisores y fuentes formales, como informales.

En general, se tiende a pensar que las personas se comportan como seres racionales, realizando permanentemente análisis de costo-beneficio cada vez que se enfrentan a información nueva. En este caso particular, respecto de cómo cuidarse y modificar conductas, o no, ante una nueva enfermedad. Sin embargo, esta percepción dista bastante del cuerpo de evidencia que ha estudiado los procesos cognitivos y conductuales ante información nueva[1].

\section{Sesgos cognitivos}

Frente a este tipo de información, los procesos cognitivos utilizan una serie de atajos, también de- nominados heurísticas, para realizar estimaciones de riesgo y así disminuir la compleja tarea de evaluar probabilidades y predecir eventos. Sin embargo, a pesar de su utilidad estos procesos de atajo suelen fallar al evaluar probabilidades y tienden a llevar a errores graves y sistemáticos[2]. Si bien los instintos nos permiten evaluar riesgos en el día a día, permitiendo ponderar mayor o menor importancia, la evidencia ha descrito que suelen ser imperfectos[3].

La situación actual de la pandemia de COVID-19 ha propuesto a la comunidad científica y a la población general, una serie de desafíos metodológicos, analíticos y reflexivos. En este caso particular, brinda también la oportunidad de reflexionar en torno a qué situaciones y procesos están relacionados al momento de incorporar nueva información basada en una aparente certeza, la consiguiente toma de decisiones y cambio conductual respecto al riesgo y la forma de comunicación del riesgo por parte de distintas fuentes, las cuales eventualmente pueden influenciar que nuestros instintos y la toma de decisiones estén muy equivocados.

Uno de los principales elementos a considerar es el sesgo de memoria[4]. Cuando se desea evaluar un riesgo, los procesos cognitivos buscan rápidamente situaciones similares y si se recuerda alguna, la percepción de riesgo suele aumentar. En el caso de la pandemia, la mayoría de las personas están o estarán expuestas a una gran cantidad de reportes de enfermos graves, hospitales colapsados y números de fallecidos sobrepasando la capacidad de los servicios mortuorios. Es de esperar que este sea el recuerdo más cercano al que el procesamiento cognitivo tenga acceso, sobre todo si anteriormente las personas han tenido alguna experiencia similar, directa o indirecta con algún familiar o conocido. La comunicación de este tipo de noticias suele describir muertes y colapsos de los sistemas, sin considerar el $97-99 \%$ de personas que logran recuperarse o que puede haber tenido cuadros leves o asintomáticos, reforzando potencialmente al sesgo de memoria.

En términos de respuesta frente al riesgo, este 
tipo de sesgos puede llevar a ambos extremos: desde un alarmismo extremo, a una complacencia indebida en personas que escucharon que todo es una exageración, o que tienen recuerdos de anuncios catastróficos del pasado que no fueron lo suficientemente graves en relación a lo comunicado[5].

Contar con la información -aunque incompletadel número bruto de contagiados, tasa de letalidad o impacto general en el acceso a atención, no basta. La forma vivencial en que se experiencia la nueva información suele ser más abrumadora que un cálculo racional.

Otro "atajo" relevante a considerar entre los procesos cognitivos frente a nueva información es la novedad, o el condicionamiento para enfocarse en amenazas nuevas[6]. Esto puede llevar a obsesionarse con reportes dramáticos e imaginar los peores escenarios, aumentando la percepción de riesgo[7]. Por ejemplo, no ponderaremos de la misma manera el riesgo de morir de influenza o de un accidente automovilístico, cuando lo comparamos con la novedosa pandemia de COVID-19 o un infrecuente viaje en avión[8].

Quizás el más poderoso de estos atajos es la emocionalidad. Los procesos cognitivos incorporan las percepciones y emociones a conclusiones racionales[9], aunque estas conclusiones contradigan los datos disponibles, debido a que ambos procesos -emocionalidad y racionalidad- están íntimamente imbricados. Las expectativas que se tienen en relación a la frecuencia de los eventos se ven tremendamente distorsionadas por el número y la intensidad emocional del contenido de los mensajes a los que las personas están expuestas[10]. En el caso particular de la pandemia, el compartir imágenes que potencialmente puedan herir la sensibilidad de las personas, como por ejemplo, muertos en las calles, bolsas de cadáveres y personal sanitario sobrepasado, aumentará la percepción de riesgo de forma sesgada[11].

A su vez, la percepción de riesgo también aumentará cuando la información que se trate de incorporar sea en mayor o menor medida incomprensible, tanto para académicos como para población general. En este caso, una nueva enfermedad de la cual aún se desconoce cómo se esparció tan rápido y donde los mismos especialistas aún no logran comprender bien su comportamiento debido a datos limitados[11]. Probablemente la novedad de la información de esta pandemia ha generado una pérdida de percepción de control y las personas han realizado intentos conductuales por retomar el control y reducir la incertidumbre mediante acciones como acaparar productos de limpieza, o retomar actividades como reuniones sociales que antes eran consideradas normales, pero ahora son de riesgo, pese a los llamados de la autoridad sanitaria a evitar estas conductas[12].

Por otro lado, están los riesgos que se toman voluntariamente o se perciben aparentemente como voluntarios, que podrían disminuir la percepción de riesgo. Por ejemplo, las personas sobreestiman el riesgo de ir a trabajar y subestiman el riesgo de salir a tomar un café o a comprar a una botillería[13]. En el comienzo de la pandemia muchas personas subestimaron el riesgo de viajar y congregarse en eventos masivos, llevando la percepción de riesgo desde un nivel insignificante respecto al riesgo real de contagio, hasta convertirlo en el mayor riesgo para la humanidad.

Aunque es relevante indicar que la percepción de riesgo no se comporta de forma racional en todos los casos, sería sumamente irresponsable dar a entender con esto que el temor al COVID-19 es completamente irracional, porque hay varias razones concretas para estar asustados. Lo correcto es reforzar una invitación permanente a realizar mejores estimaciones y respuestas tanto conductuales como sociales, al conocer los sesgos que afectan la ponderación de riesgo. La amenaza real para cada individuo no es la estadística de la amenaza, sino la percepción de riesgo. Los procesos cognitivos suelen redondear las probabilidades de riesgo a "casi cero" y tanto la conducta paralizante de no realizar nada, como enfocarse en el peor escenario implican sobrerreacción al hecho concreto.

En términos sociales, cuando una cantidad importante de personas se enfoca en el peor de los escenarios, reforzado por la forma en que la prensa y los medios formales e informales comunican los hechos y sumado a una desconfianza contemporánea en la autoridad y las fuentes de información formales, se obtiene la receta perfecta para la percepción social de pérdida de control, pérdida de gobernabilidad y pánico colectivo. Del mismo modo, si un grupo importante de personas desprecian la amenaza y creen que es una exageración, deslegitiman las fuentes formales de información e imponen ideologías que minimizan el riesgo real, el resultado pareciera ser el mismo. Luego de haber revisado los principales sesgos cognitivos que operarían en una amenaza de riesgo global, es necesario revisar también las características del mensaje.

\section{Factores de confusión}

Consideremos que toda la información viene de fuentes que en general se estiman formales para comunicar riesgo en el caso de una pandemia, como por 
ejemplo, médicos, estadísticos y epidemiólogos, pero también se incluyen ingenieros, sociólogos o básicamente cualquier persona que tenga habilidades mínimas de análisis para construir un gráfico con una curva que se ajuste a los sesgos señalados anteriormente, con el fin de disminuir la percepción de incertidumbre. Frente a cualquier gráfico o tabla basada en la estadística, se podría argumentar que "hay que dejar que los datos hablen". Sin embargo, quienes comunican los datos realizan la interpretación por nosotros.

Hay una serie de problemas relacionados con este punto: En primer lugar, las hipótesis estadísticas no son lo mismo que las hipótesis experimentales. Cualquier conjunto de datos puede ser utilizado para realizar hipótesis estadísticas, puesto que éstas solo describen y analizan conjuntos de datos. Las hipótesis experimentales implican un nivel de abstracción mayor y se enfocan en comprender la naturaleza de un fenómeno y generar nuevas hipótesis. En segundo lugar, se supone a priori que los expertos anteriormente señalados estarán libres de los sesgos cognitivos que acabamos de revisar, pero se ha documentado extensamente los conflictos de interés que suelen tener quienes comunican análisis por su cuenta y lo cierto es que también toman decisiones respecto a qué datos incluir, los parámetros, los supuestos dispuestos a aceptar y la forma de interpretar y presentar los resultados[14]. Por último, es necesario considerar otras variables que pueden generar que las conclusiones y predicciones sean erróneas.

Supongamos que se requiere estimar el número de personas que están enfermas en una ciudad, pero sólo se tiene información de las que están hospitalizadas. Si el 15\% de los hospitalizados tienen la enfermedad, debido al sesgo de selección de la muestra -población clínica no necesariamente es representativa de la población general-, se obtendrá una sobreestimación de los enfermos en la ciudad. Si a todos los pacientes que se les hace el test son los sintomáticos respiratorios que además están hospitalizados, se sesgará la estimación al no considerar las variables y características de los asintomáticos, a los sintomáticos leves, a los sintomáticos moderados/graves que no se hospitalizan y a los graves que fallecen antes de acceder a una hospitalización[15].

Esto puede explicar, en parte, las grandes diferencias que existen entre distintos países en las tasas de letalidad, donde puede haber diferencias de un orden de magnitud. Eventualmente, países que mantienen una estrategia de hacer tests a todos sus ciudadanos tendrán una tasa de contagio muy alta -casos incidentes acumulados- pero una letalidad baja. Por su parte, si sólo se hacen tests a los pacientes más graves, la tasa de contagio será relativamente baja comparada con el ejemplo anterior, pero la letalidad será mucho más alta. Por último, en países donde no existe la posibilidad de hacer tests, la tasa de contagio y la letalidad van a ser nulas, aunque puede aumentar la tasa de mortalidad general del país. Uno de los valores que más se repitió del riesgo de COVID-19 era una letalidad de 3,4\% reportado por la OMS a comienzos de marzo del año 2020[16]. Esta estimación se concentraba en los pacientes más graves, ignoraba las infecciones no detectadas y la fuerte asociación de la enfermedad con la edad. Por ejemplo, en el caso de los pasajeros del crucero Diamond Princess - una cohorte cerrada de edad avanzada-, la letalidad fue de $1 \%$, por lo que es probable que la letalidad en la población general sea mucho menor. Por su parte, en los países que han realizado más pruebas -Corea del Sur y Alemania-, la tasa es $0,7 \%$ y $0,2 \%$, respectivamente[17]. Incluso en estos países, muchos casos no son diagnosticados, por lo que se podría anticipar una tasa aún menor. La tasa de letalidad en Chile a la fecha en que se escribió este artículo, es 1,05\%, la cual se puede explicar en parte, por un gran número de tests en pacientes con síntomas leves y una limitación en el número de tests que pueden ser procesados y notificados[18]. No existen muchas razones para pensar que la enfermedad se comporta en forma diferente considerando solo los límites geográficos como variable relevante.

Una manera de mejorar el origen de los datos con los que se realizan estos análisis, y así realizar una estimación menos sesgada y más precisa del número de contagiados y de letalidad, es mediante muestreo aleatorio a la población general, junto con la realización de exámenes a todos los fallecidos, independiente de la causa de muerte. Sin embargo, desde un punto de vista logístico, es muy difícil y hasta la fecha no hay países que ocupen ambas estrategias. Es necesario que estas limitaciones originadas del sesgo de selección sean presentadas o transparentadas como supuestos al momento de comunicar cualquier resultado.

También se debe considerar la distinta precisión de los tests incluida en el error aleatorio o no sistemático, ya que la precisión de cada test no está estandarizada y depende de variables como el tipo de reactivo, la calidad de la muestra y la carga viral del paciente. Un test con sensibilidad de $60 \%$ deja a un $40 \%$ de contagiados sin diagnosticar y si se hace un test de confirmación, la proporción de falsos negativos es aún más alta[19].

Otras variables que pueden influir en la estimación de resultados son el porcentaje de ancianos en 
determinada población y la forma en que se reportan las muertes. Por ejemplo, en algunos países, cualquier persona que fallezca luego de haberse diagnosticado como COVID-19, se considera deceso por esa causa, incluso si no fue la causa de muerte. A la inversa, muchos pacientes pueden fallecer a causa del virus sin que hayan sido diagnosticados[15]. Llama la atención que los muertos COVID-19 sean relativamente pocos cuando se compara con la mortalidad general esperada que es 10 o 20 veces mayor. En Chile, fallecen aproximadamente 105.000 personas al año (aproximadamente 288 fallecidos/día) con una proporción de muertos COVID-19, en la fecha que se escribió este artículo, de solo 1,05\% calculado desde el primer fallecido [20],[21]. Esto puede orientar a un subreporte de la mortalidad por la pandemia, pero también a una sobreestimación de la gravedad de ésta. Estas diferencias en la metodología de la extracción de los datos es la que hace que las comparaciones entre diferentes países o regiones sea inconsecuente

Los tres principales confundentes descritos anteriormente pueden llevar a subestimar o sobrestimar los resultados en medio de una pandemia, pero hay otros elementos a considerar en la comunicación de los resultados: la forma en que se realizan y analizan los modelos.

En general, los modelos predictivos que analizan sólo una variable, por ejemplo, días de lockdown, tiempo entre el primer contagio y la primera muerte, programa de vacunación, entre otras, tienden más a equivocarse que a acertar en sus proyecciones. Debido a que no suelen considerar otras variables, a saber: edad estratificada de la población, idiosincrasia local y cultural, estación y época del año, metros cuadrados de superficie habitable per cápita, capacidad de los sistemas de salud que varía a lo largo del tiempo de forma no estática, nivel de escolaridad, ingreso per cápita, temperaturas extremas, porcentaje de pobreza, nivel socioeconómico y un largo etcétera. Además, existen muchas otras variables que no son incorporadas a los modelos de predicción porque se les da poca importancia o ni siquiera se ha estudiado que se comporten como tales. La influencia de todas estas variables hace que los modelos predictivos no sean válidos, ya que sólo analizan una o pocas variables, desconociendo la influencia de las otras.

\section{Discusión}

Uno de los objetos de estudio de la epidemiología es estudiar las probabilidades de ocurrencia de distintos eventos o condiciones. Sin embargo, uno de los desafíos más importantes y complejos es el de comunicar la incertidumbre de los análisis que se han obtenido producto de decisiones, sesgos y calidad del origen de los datos, además de comunicar esta incertidumbre en un lenguaje que sea comprensible para la población general.

Hasta el momento, pareciera ser que la situación actual dista mucho de eso, debido a que los hechos no se están midiendo correctamente, por lo que los datos se encuentran incompletos o en muchos casos no existen. Los datos con los que se cuentan se miden en forma no estandarizada y con muchos sesgos, lo que no permite realizar análisis de comparaciones con datos de otros países[15]. Tampoco se cuenta con un nivel adecuado de transparencia hacia la sociedad civil y científica por parte de las autoridades en relación a evolución de contagios, causas de muerte general, expectativas de atención sanitaria y limitaciones logísticas para entregar datos confiables[22]. Cuando la calidad de los datos es mala, no podemos tener confianza en las inferencias que se hagan.

Los encargados de comunicar los hechos y análisis sobre COVID-19 no han podido demostrar el suficiente sustento técnico para transmitir a equipos sanitarios, académicos y población general el nivel de incertidumbre asociada a los datos y los pronósticos[23]. Tampoco se han descrito los supuestos de los modelos de predicciones. Al momento de realizar estos modelos, no se incluyen variables relevantes que actúan como potenciales confundentes[24]. Por último, al entregar los resultados, se reporta sólo la estimación central. En algunos casos incluso, como mejor o peor escenario. Los resultados deben ser entregados como rango y con énfasis en la precisión[25].

Los medios de comunicación formal y periodistas no cotejan la información que reciben con otros expertos, antes de difundirla. Los titulares no reflejan la información correcta y generalmente la presentan de forma parcial y sensacionalista. Muchas veces recurren, para la difusión de datos, a personas sin formación en epidemiología o enfermedades infecciosas y dan gran cobertura a escenarios no realistas y sesgados. También se centran en eventos de más alto impacto emocional o situaciones que son anecdóticas en el contexto general [26]-[29].

Finalmente, estas comunicaciones desde autoridades y medios de comunicación llegan a la población general, quienes reciben esta información de manera parcial y la interpretan en función de sesgos y temores individuales propios de la percepción del riesgo, sin dejar un espacio para el análisis crítico, lo que se traduce, finalmente, en conductas irracionales en cualquiera de los dos extremos[2]. En muchos 
casos, se pierde incluso el primer filtro de los medios de comunicación formal, pues la penetración de las redes sociales permite el consumo inmediato de análisis crudos y noticias falsas. En términos del poder que ejerce la academia, si bien la mayoría de la población general puede identificar un charlatán, como el que dice en medios de comunicación formal que hay que tomar jugo de limón en la mañana, despertar y hacer saludos al sol como intervenciones para evitar contagios, el cuestionar a médicos, ingenieros o economistas es más complejo cuando éstos muestran un gráfico aparentemente objetivo, aunque ninguno de estos profesionales tenga las credenciales necesarias para entregar información adecuada a partir del mismo.

El escenario de pandemia mundial puede ser transformado en una oportunidad de plantear la idea que, a largo plazo, existan esfuerzos educacionales y comunicacionales para preparar a la población y desarrollar el pensamiento crítico en la búsqueda de información. Esta educación debiera idealmente incluir rudimentos estadísticos que permitan transformar a consumidores pasivos de información en interlocutores activos que comprendan las limitaciones e incertidumbres propias de los datos transmitidos y sean capaces de analizarlos y seleccionarlos. Sin duda, hacer un llamado a autoridades académicas y gubernamentales es un primer paso, pero implementar estrategias para mejorar el acceso a información de alta calidad en salud y desarrollar estrategias comunicacionales con foco en la educación y desarrollo de pensamiento crítico podría potencialmente ser una gran inversión en Salud Pública.

\section{Financiamiento}

Los autores declaran que no hubo financiamiento.

\section{Conflicto de intereses}

Los autores no reportan conflictos de interés.

\section{Referencias}

1. Gilovich T, Griffin D, Kahneman $D$, editors. Heuristics and Biases. Cambridge University Press; 2002 Jul 8; http:// dx.doi.org/10.1017/ cbo9780511808098

2. Tversky A, Kahneman D. Judgment under Uncertainty: heuristics and Biases. Science. 1974 Sep;185(4157):1124-31. https://doi.org/10.1126/ science.185.4157.1124 PMID:17835457

3. Marewski JN, Gigerenzer G. Heuristic decision making in medicine. Dialogues Clin Neurosci. 2012 Mar;14(1):7789. PMID:22577307

4. Madan CR, Ludvig EA, Spetch $\mathrm{ML}$. Remembering the best and worst of times: memories for extreme outcomes bias risky decisions. Psychon Bull Rev. 2014 Jun;21(3):629-36. https:// doi.org/10.3758/s13423-0130542-9 PMID:24189991

5. Slovic P. Perception of risk. Science. 1987
Apr;236(4799):280-5.

https://doi.org/10.1126/

science.3563507 PMID:3563507

6. Van Boven L, White K, Huber

M. Immediacy bias in emotion perception: current emotions seem more intense than previous emotions. J Exp Psychol Gen. 2009 Aug;138(3):368-82. https://doi.org/10.1037/ a0016074 PMID:19653796

7. Persaud N, Heneghan C. Novelty Bias Catalogue of Bias. https:// catalogofbias.org/biases/noveltybias/

8. Schwartz O, Hsu A, Dayan $P$. Space and time in visual context. Nat Rev Neurosci. 2007 Jul;8(7):522-35. https:// doi.org/10.1038/nrn2155 PMID:17585305

9. Sackett DL. Bias in analytic research. J Chronic Dis. 1979;32(1-2):51-63. https:// doi.org/10.1016/00219681(79)90012-2 PMID:447779

10. Kahneman D. Thinking, fast and slow. 1st ed. New York: Farrar, Straus and Giroux; 2011. 499 p. p.
11. Fischhoff B, Gonzalez RM, Lerner JS, Small DA. Evolving judgments of terror risks: foresight, hindsight, and emotion. J Exp Psychol Appl. 2005 Jun;11(2):124-39. https://doi.org/10.1037/1076898X.11.2.124 PMID:15998184

12. López-Vázquez E, Marván ML. Risk perception, stress and coping strategies in two catastrphe risk situations. Soc Behav Personal. 2003;31(1):6170. https://doi.org/10.2224/ sbp.2003.31.1.61.

13. Barnett J, Breakwell GM. Risk perception and experience: hazard personality profiles and individual differences. Risk Anal. 2001 Feb;21(1):171-7. https://doi.org/10.1111/02724332.211099 PMID:11332545

14. Marcovitch $H$, Barbour $V$, Borrell C, Bosch F, Fernández E, Macdonald $\mathrm{H}$, et al.; Esteve Foundation Discussion Group. Conflict of interest in science communication: more than a financial issue. Report from Esteve Foundation Discussion 
Group, April 2009. Croat Med J. 2010 Feb;51(1):7-15. https:// doi.org/10.3325/cmj.2010.51.7 PMID:20162740

15. Lipsitch M, Donnelly CA, Fraser C, Blake IM, Cori A, Dorigatti

I, et al. Potential Biases in Estimating Absolute and Relative Case-Fatality Risks during Outbreaks. PLoS Negl Trop Dis. 2015 Jul;9(7):e0003846. https://doi.org/10.1371/journal. pntd.0003846 PMID:26181387

16. [Available from: https://www. who.int/dg/speeches/detail/ who-director-general-s-openingremarks-at-the-media-briefingon-covid-19---3-march-2020

17. Jordans F. Experts: Rapid testing helps explain few German virus deaths 2020 [Available from: https://apnews.com/ ad9a6af47c3b55fd83080 c9168afaaf4

18. Comunicaciones-MSGG Sd. Cifras oficiales COVID-19 Gob. cl2020 [Available from: https:// www.gob.cl/coronavirus/ cifrasoficiales/\#reportes

19. Marshall R. The Predictive Value of Simple Rules for Combining Two Diagnostic Tests. Biometrics. 1989;45(4):1213-22. https://doi. org/10.2307/2531772.

20. Defunciones y Mortalidad
General según región y comuna de residencia. Chile, Año 2016 2016 [Available from: http:// www.deis.cl/wp-content/2017/ gobCL-sitios-1.0/assets/ SerieDefunciones_2000_2015. html

21. Casos confirmados en Chile COVID-19 2020: https://www. minsal.cl/nuevo-coronavirus2019-ncov/casos-confirmadosen-chile-covid-19/

22. Aguilera $X$. La decisión de las cuarentenas y los detalles del Plan Económico de Emergencia. In: Vergara N, Del Rio M, Saavedra $C$, editors. Hablemos en off. 2020.

23. Gorelick MH. Bias arising from missing data in predictive models. J Clin Epidemiol. 2006 Oct;59(10):1115-23. https://doi.org/10.1016/j. jclinepi.2004.11.029 PMID:16980153

24. Goodson M. All models are wrong, but some are completely wrong Royal Statistical Society2020 [Available from: https://rssdss.design. blog/2020/03/31/all-modelsare-wrong-but-some-arecompletely-wrong/.

25. Wolff RF, Moons KG, Riley RD, Whiting PF, Westwood M, Collins GS, et al.; PROBAST
Groupt. PROBAST: A Tool to Assess the Risk of Bias and Applicability of Prediction Model Studies. Ann Intern Med. 2019 Jan;170(1):51-8. https:// doi.org/10.7326/M18-1376 PMID:30596875

26. Intendente Guevara señala que hay tres denuncias por personas que salieron en aeronaves desde la RM rumbo a segundas viviendas. La Tercera. 2020.

27. Muñoz A. Big Data en tiempos de pandemia: Cómo podría ayudar y sus límites en la emergencia. La Tercera. 2020 March25, 2020.

28. Uranga N. Cuántos contagiados por Covid-19 tenía cada país al momento de registrar su primera muerte. El Mercurio. 2020 March 20, 2020.

29. cooperativa.cl. Coronavirus: Investigadores estiman que sin cuarentenas zonales Santiago superaría los 100 mil casos en junio 2020 [Available from: https://www.cooperativa. cl/noticias/sociedad/salud/ coronavirus/coronavirusinvestigadores-estiman-quesin-cuarentenas-zonalessantiago/2020-04-09/131636. html

30. Casos confirmados en Chile COVID-19. 2020. 